\title{
EDUCANDO PARA O DIAGNÓSTICO PRECOCE DA HANSENÍASE NO MUNICÍPIO DE RONDONÓPOLIS- MATO GROSSO
}

\author{
Débora Aparecida da Silva Santos \\ Universidade Federal de Mato Grosso \\ deboraassantos@hotmail.com \\ Liliam Carla Vieira Gimenes Silva \\ Universidade Federal de Mato Grosso \\ liliamcarla@hotmail.com
}

\author{
Laura Bordognon Spessatto \\ Universidade Federal de Mato Grosso \\ lauraspessatto@hotmail.com \\ Luan Sudário Melo \\ Universidade Federal de Mato Grosso \\ luansudario@hotmail.com \\ Lourenço Ribeiro da Cruz Neto \\ Secretaria Municipal de Saúde de Rondonópolis-MT \\ lookruz@hotmail.com
}

\begin{abstract}
Resumo
Rondonópolis-MT vem apresentando altos índices de casos de hanseníase, levando a necessidade de ações educativas preventivas. O projeto de extensão "Hanseníase: diagnóstico precoce é a solução" é realizado com parceria da Secretaria de Saúde há 4 anos com o Curso de Enfermagem UFMT/ICEN/CUR. Este artigo objetivou descrever a realização das atividades de orientação sobre prevenção da hanseníase nas áreas das unidades de saúde, educação em serviço, palestras em escolas e participação em mutirões, entre março de 2014 a março de 2015. O público-alvo foram 1764 moradores, além dos alunos e profissionais das escolas e equipes de saúde. Foram encaminhados 22 casos suspeitos, sendo confirmados 11 novos, formação de profissionais e nas escolas. Nesta ação de cidadania, o projeto contribuiu significativamente com o diagnóstico precoce da hanseníase e com a saúde pública do município. Sugere-se que novos projetos sejam realizados para favorecer a qualidade da atenção básica, estimulando a participação da academia através da extensãouniversitária.

Palavras-chaves: Educação em saúde. Hanseníase. Diagnóstico precoce. Enfermagem. Promoção em saúde.
\end{abstract}

\section{EDUCATING FOR EARLY DIAGNOSIS OF LEPROSY IN THE MUNICIPALITY OF MATO GROSSO RONDONÓPOLIS}

\begin{abstract}
Rondonópolis-MT has shown high rates of leprosy cases, leading to the need for preventive educational activities. The extension project "Leprosy: Early diagnosis is the solution" is carried out in partnership with the Health Department for 4 years with the Nursing Course UFMT / ICEN / CUR. This article aimed to describe the realization of educational activities on prevention of leprosy in the areas of health facilities, in-service education, lectures in schools and participation in joint efforts between March 2014 and March 2015. The audience were 1764 residents, in addition to students and professionals schools and health teams. They were referred 22 suspected cases and confirmed 11 new, professional training and schools. This citizenship action, the project significantly contributed to the early diagnosis of leprosy and the public health of the city. It is suggested that new projects are undertaken to promote the quality of primary care, encouraging the participation of academia through the university extension.
\end{abstract}

Keywords: Health education. Leprosy. Early Diagnosis. Nursing. Health promotion.

\section{EDUCANDO PARA EL DIAGNÓSTICO PRECOZ DE LA HANSENÍASE EN EL MUNICIPIO DE RONDONÓPOLIS - MATO GROSSO}

\section{Resumen}

En la municipalidad de Rondonópolis, Mato Grosso ocurren elevados índices de casos de lepra, lo que lleva a la necesidad de realizar actividades educativas preventivas. El proyecto de extensión "Lepra: El diagnóstico precoz es la solución" se llevó a cabo en colaboración con el Departamento de Salud hace cuatro años en el Curso de Enfermería de la UFMT/ICEN/CUR. Este artículo tiene como objetivo describir la realización de actividades educativas sobre la prevención de la lepra en las áreas de los servicios de salud, educación, conferencias en las escuelas y la participación en los esfuerzos conjuntos entre marzo de 2014 y marzo de 2015. El público atendido fueron 1764 residentes, además de la participación de estudiantes y profesionales de escuelas y equipos de salud. Fueron referidos 22 casos sospechosos y 
confirmados 11 nuevos. En esta acción de ciudadanía, el proyecto ha contribuido significativamente al diagnóstico precoz de la lepra y la salud pública de la ciudad. Se sugiere que se lleven a cabo nuevos proyectos para promover la calidad de la atención primaria, fomentando la participación de la academia a través de la extensión universitaria.

Palabras clave: Educación para la salud. La lepra. El diagnóstico precoz. Enfermería. Promoción de la salud. 
Educando para o diagnóstico precoce da hanseníase no município de Rondonópolis- Mato Grosso

\section{INTRODUÇÃO}

A hanseníase é uma doença infectocontagiosa de evolução lenta, causada pelo Mycobacterium leprae conhecido como Bacilo de Hansen. O agente etiológico é considerado um parasita intracelular obrigatório, possuindo afinidade com células cutâneas e nervos periféricos, instalando no organismo da pessoa infectada e podendo se multiplicar. Manifesta-se através do surgimento de sinais e sintomas dermatoneurológicos como manchas pigmentares ou discrômicas, placa individual ou em aglomerados, infiltrações, pápulas e/ou nódulos. Essas lesões localizam-se em qualquer região do corpo, porém ocorrem com maior frequência na face, nas orelhas, nas nádegas, nos braços, nas pernas e nas costas. A sensibilidade nas áreas lesionadas pode estar reduzida (hipoestesia), ausente (anestesia) ou aumentada (hiperestesia) (BRASIL, 2002).

Esse microrganismo possui a capacidade de infectar uma grande quantidade de pessoas, caracterizando alta infectividade, no entanto somente algumas pessoas adoecem, pois possui baixa patogenicidade. Essas propriedades são em função das suas características intrínsecas, mas que dependem, sobretudo, da relação parasita-hospedeiro e o grau de endemicidade do meio, entre outros aspectos (BRASIL, 2010).

A principal via de eliminação do bacilo pelo doente e a porta de entrada no organismo passível de ser infectado são as vias aéreas, no entanto para que a transmissão aconteça é necessário contato direto com o doente não tratado. $\mathrm{O}$ aparecimento das manifestações clínicas na pessoa infectada pelo bacilo pode ocorrer após um longo período de incubação, de 2 a 7 anos (BRASIL, 2002).

Esta patologia tem importante relevância para a saúde pública, devido sua magnitude e seu alto poder de causar incapacidades, atingindo principalmente pessoas em faixa etária econômica ativa comprometendo seu desenvolvimento na sociedade. $O$ seu alto potencial incapacitante está diretamente ligado à capacidade do microrganismo causador da doença penetrar na célula nervosa e também ao seu poder imunogênico (BRASIL, 2009). As taxas mais altas de prevalência e incidência pertencem ao Brasil, com $87 \%$ em relação aos números notificados na Américas, ganhando a segunda posição no mundo. Acredita-se que esses resultados estão diretamente relacionados à grande identificação e notificação ou realmente por altos índices de casos (MARTINS; CAPONI, 2010). No Brasil a hanseníase apresenta tendência de estabilidade nos índices de detecção, mas nas regiões do Norte, Centro-Oeste e Nordeste, a situação ainda é alarmante. Nestas regiões que alcançam números altos de detecções e de 
Educando para o diagnóstico precoce da hanseníase no município de Rondonópolis- Mato Grosso

crescimento da doença, supõe-se que os resultados da grande vulnerabilidade estão diretamente ligados à geografia desses locais (BRASIL, 2008a).

O estado de Mato Grosso (MT) está entre os estados que mais apresenta casos de hanseníase. A forte relação da doença iniciou a partir dos movimentos migratórios que cresceram em busca da parcela da riqueza de uma economia agrícola e desenvolvimento urbano que permanecem em crescimento. Esse processo altera a estrutura epidemiológica, pois o estado possui áreas extensas de matas e floresta sendo grande atrativo. Essas áreas "desocupadas" que produzem mudanças na existência e expansão de doenças infecciosas e parasitárias, como a hanseníase. Rondonópolis desde o início da migração e com investimentos do Governo Federal que fez duplicar sua população esteve entre as cidades do estado com grandes números de casos de hanseníase e, por tanto, mostra-se como foco histórico da doença, permanecendo até a atualidade (MAGALHÃES et al. 2011).

Rondonópolis (MT) apresenta coeficiente de números de casos acima do preconizado pela Organização Mundial de Saúde (OMS) e pelo Ministério da Saúde (MS). O serviço de saúde oferece um Centro de Referência com estrutura de atendimento especializado desde 1883 e é composto por uma equipe de profissionais especializados e treinados como dermatologista e hansenólogo e enfermeira especialista em saúde pública. O gestor ciente do problema de saúde pública que persiste, tem como proposta facilitar a realização dos exames para diagnósticos precisos e suporte aos profissionais que atuam na rede básica (GOVERNO MUNICIPAL DE RONDONÓPOLIS, 2011).

O diagnóstico da hanseníase pode ser feito através de diagnóstico clínico buscando os sinais dermatológicos e neurológicos da doença, testes de sensibilidade e diagnóstico laboratorial. No diagnóstico clínico é realizada a anamnese, momento de coleta de informações sobre história clínica (sinais e sintomas) e epidemiológica (fonte de infecção). Logo é realizado o exame físico com avaliação dermatoneurológica, com o intuito de identificar sinais clínicos da doença. A condição de vida é fator importante relacionado à transmissão, os níveis de endemia e as condições socioeconômicas desfavoráveis, assim como condições precárias de vida e elevado número de pessoas convivendo em um mesmo ambiente, influem no risco de adoecer (BRASIL, 2002). O contato direto e prolongado com a pessoa infectada em local fechado, com pouca ventilação e pouca iluminação solar, aumenta a chance de infecção (BRASIL, 2008b).

De acordo com o Ministério da Saúde, através dos dados do Sistema de Informação de Atenção Básica, Rondonópolis (MT) em 2014 possuiu 1016 casos de hanseníase cadastrados e 979 casos acompanhados mensalmente através das unidades de saúde de atenção básica do município e do serviço de referência (MINISTÉRIO DA SAÙDE, 2015). Estes dados sugerem 
Educando para o diagnóstico precoce da hanseníase no município de Rondonópolis- Mato Grosso

que neste município a hanseníase deve ser considerada como um doença de importante controle, cabendo as equipes interdisciplinares a realização de diagnóstico precoce e tratamento adequado.

Neste sentido, o diagnóstico precoce da hanseníase nos municípios, é indispensável ao controle da incidência desta doença na população. Além dos serviços de saúde da atenção básica, cabe a universidade, através de docentes e discentes, contribuir para o conhecimento sobre a prevenção, além de produzir ações que possam facilitar o diagnóstico precoce, contribuindo com tratamento adequado e com a cura desta doença.

Por meio da extensão, a universidade tem a oportunidade de levar, até a comunidade, os conhecimentos de que é detentora, os novos conhecimentos que produz com a pesquisa, e que normalmente divulga como ensino. É uma forma da universidade socializar e democratizar o conhecimento, levando-o aos não universitários e sendo difundido, mantendo-se consoante aos próprios interesses da comunidade (SILVA, 1997).

Neste sentido, o objetivo geral deste artigo foi descrever as ações do projeto de extensão no que tange as atividades de treinamento em serviço para os profissionais de saúde, visitas domiciliárias junto com a (o) enfermeira (o) da unidade, mutirão, orientações sobre a prevenção da hanseníase e educação em saúde nas escolas das áreas de abrangência das unidades de saúde no município de Rondonópolis (MT).

Além disso, dentre as ações deste projeto de extensão, através do treinamento em serviço, teve como objetivos:propiciar conhecimento teórico-científico sobre hanseníase aos Agentes Comunitários de Saúde das unidades; reforçar o conhecimento na identificação de sinais e sintomas; ressaltar a importância do diagnóstico precoce e tratamento sem interrupção. Através das visitas domiciliárias: realizar busca ativa e avaliação dos comunicantes; informar aos usuários sobre a importância em procurar as unidades de atendimentos, no caso de suspeita ou sintomas da doença e esclarecer sobre sinais e sintomas, formas de contaminação, tratamento e prevenção da hanseníase. Através dos mutirões: realizar busca ativa de casos novos através do exame de pele; viabilizar o processo de identificação dos sinais e sintomas da doença e informar sobre a importância do tratamento no controle da doença. Através da educação em saúde: levar ao conhecimento do público um sucinto histórico e processo evolutivo da hanseníase no município, para tentar diminuir o estigma e preconceito; informarsobre a importância em procurar as unidades de atendimentos, no caso de suspeita ou sintomas e fornecer informações corretas sobre formas de contaminação, sinais e sintomas, tratamento e prevenção e divulgar as ações da Secretaria Municipal de Saúde em relação ao controle e tratamento da hanseníase em Rondonópolis (MT). 
Educando para o diagnóstico precoce da hanseníase no município de Rondonópolis- Mato Grosso

\section{MATERIAIS E MÉTODOS}

Para o desenvolvimento deste artigo sobre as ações do projeto de extensão, foi realizada a busca de material científico sobre hanseníase, pesquisa e coleta de dados da situação dos casos ocorridos no município na Secretaria Municipal de Saúde (SMS) de Rondonópolis (MT). Este projeto foi cadastrado e autorizado pelas instâncias responsáveis pelos projetos de extensões da Universidade Federal de Mato Grosso (UFMT), Campus Universitário de Rondonópolis (CUR).

O projeto foi desenvolvido nas unidades de Estratégia em Saúde da Família (ESF) e nas escolas das áreas de abrangência destas unidades do município entre o período de março de 2014 à março de 2015. Além disso, foram realizados pela SMS alguns eventos a níveis nacional e municipal que contaram coma participação deste projeto. O público-alvo foram os moradores dos bairros que compreendem as unidades de ESF, que tiveram interesse em conhecer mais sobre a Hanseníase ou que apresentaram qualquer sinal ou sintoma que caracterize a Doença de Hansen, seja no domicílio ou nas unidades. As ações foram desenvolvidas pelos executores do projeto em parceria com a SMS e realizadas nas unidades de ESF de acordo com a programação planejada pela SMS.

As atividades foram realizadas semanalmente nas unidades de ESF durante três dias da seguinte forma: $1^{\circ}$ dia: treinamento em serviço, realizado pelo enfermeiro da SMS responsável pelo Programa de Hanseníase, Tuberculose e Saúde Prisional; $2^{\circ}$ dia: visitas domiciliárias realizadas pelo enfermeiro da SMS acompanhado pela (o) enfermeira (o) da unidade ESF; $3^{\circ}$ dia: mutirões realizados para a busca ativa de casos novos através do exame de pele com teste de sensibilidade com estesiômetro e orientações.

Além disso, foi desenvolvida educação em saúde nas escolas de abrangência das unidades de ESF, pelos executores do projeto, executada seguindo as seguintes etapas: Etapa 1: Dinâmica de Acolhimento: sensibilização para o trabalho e conhecimento do grupo através da dinâmica “Estigma e Preconceitos"; Etapa 2: Palestra: Informações e discussão dos conceitos básicos sobre a temática abordada com a apresentação por data show de resumos sobre sinais e sintomas, formas clínicas, transmissão, diagnóstico, tratamento e principais dúvidas sobre a hanseníase. Além disso, para melhor compreensão dos ouvintes, foram demonstradas imagens das principais formas clínicas da Hanseníase; Etapa 3: Dinâmica de Afastamento: realização da dinâmica 
Educando para o diagnóstico precoce da hanseníase no município de Rondonópolis- Mato Grosso

"Praticando a sensibilidade", com a finalidade de conscientizar sobre a incidência elevada da hanseníase e necessidade de realização de exames para o diagnóstico precoce; Etapa 4: para finalizar, foi aplicado um questionário para avaliação das técnicas utilizadas na execução do projeto e algumas dúvidas foram sanadas de maneira individual com o intuito de avaliar o trabalho apresentado e as técnicas utilizadas para abordagem do tema.

A divulgação das ações realizadas nas unidades de ESF e da educação em saúde foram realizadas em parceria com as Agentes Comunitárias de Saúde (ACS), além da entrega de convites, divulgação da campanha com cartazes nos pontos comerciais do bairro, divulgação durante a semana que precedeu as atividades, distribuição de panfletos sobre a Hanseníase e exposição de cartazes e álbum seriado na ESF. O material de mobilização foi confeccionado pelas executoras do projeto. Todas estas atividades desenvolvidas estão descritas neste artigo.

\section{RESULTADOS E ANÁLISES}

A equipe executora deste projeto de extensão participou de 03 eventos nacionais e municipais e 09 mutirões, onde 2.189 pessoas foram atendidas. Nos eventos, obteve-se a participação de moradores dos bairros do município e região. Já nos mutirões, observou-se a participação dos usuários dos serviços de saúde da área de abrangência da comunidade das unidades de saúde. Durantes os eventos e mutirões foram realizadas orientações e exames de pele, sendo que os casos identificados como suspeitos foram encaminhados para atendimento especializado na unidade de referência do município.

Silva \& Paz (2010) buscaram compreender o fenômeno das atividades de educação em saúde no Programa de Controle da Hanseníase realizadas nos serviços de atenção básica no município de Rio de Janeiro, demonstrando que é essencial a participação dos usuários dos serviços em atividades educativas e que o profissional de saúde considera que deve informar o doente, os comunicantes sobre a hanseníase e que esta ação auxilia a comunidade.

Dentre os eventos, podem ser citados: "Dia Mundial da Hanseníase” realizado na Praça Brasil atendeu 116 pessoas (5,3\%); "Mutirão de ações de promoção à saúde" no Posto Masut, com total de 25 atendimentos (1,1\%); e a Ação Global atendidas 12 pessoas (0,5\%). Nas unidades de Estratégia de Saúde da Família (ESF), os mutirões aconteceram aos sábados, com início às $8 \mathrm{~h}$ e término às $12 \mathrm{~h}$. Na unidade de ESF Cidade de Deus foram atendidas 39 pessoas (1,8\%); na ESF Cidade Alta 07 pessoas (0,3\%); no Centro de Saúde Nossa Senhora do Amparo, 42 pessoas (1,9\%); na ESF Parque Industrial, 20 pessoas atendidas (0,9\%) e na ESF Vila Paulista, 
Educando para o diagnóstico precoce da hanseníase no município de Rondonópolis- Mato Grosso

25 pessoas $(1,1 \%)$. Além disso, foram atendidas pessoas em instituições não governamentais de assistência a pessoas carentes como a Casa Esperança, com um total 50 pessoas atendidas (2,4\%) e no Lar Cristão, 89 pessoas (4,1\%). Durante a realização dos eventos e mutirões foi observada maior participação de pessoas do sexo feminino com total de 247 pessoas 88,7\% (Figura 1).

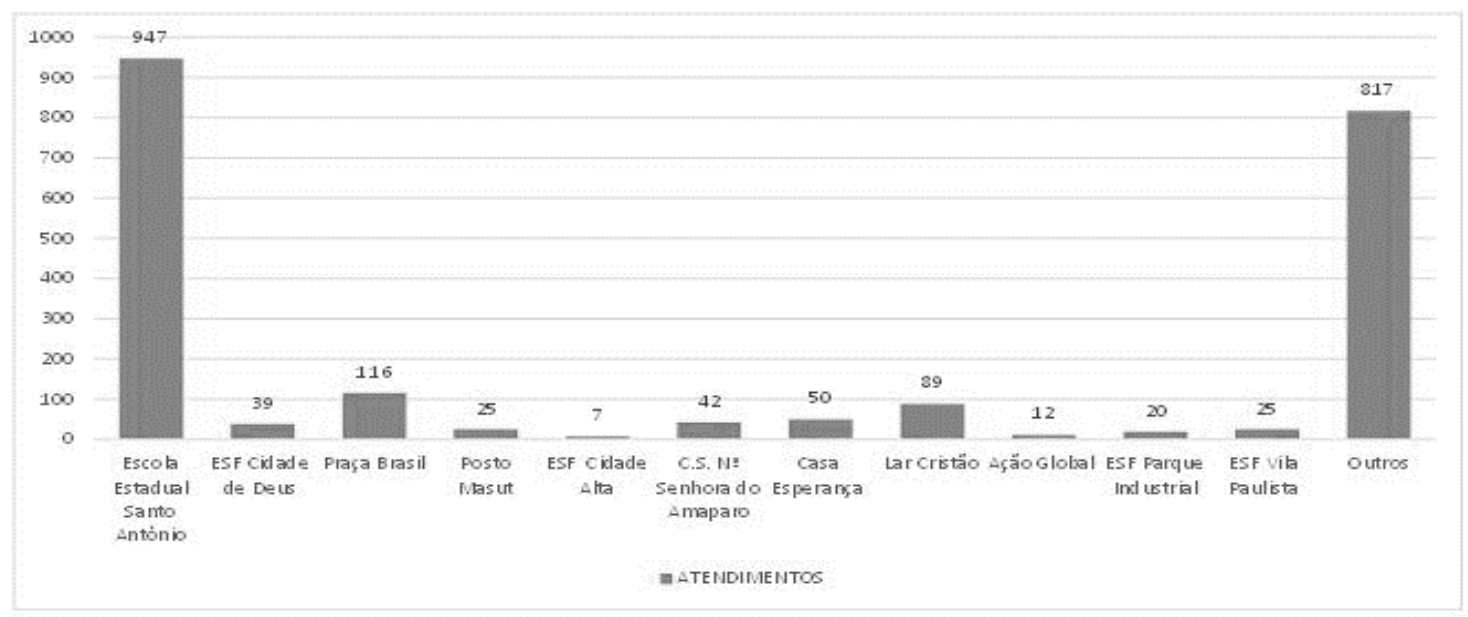

Figura 1: Participação de pessoas nos eventos do Projeto de Extensão Hanseníase: diagnóstico precoce é a solução. Rondonópolis (MT), 2015.

Um estudo ecológico de análise espacial da hanseníase dos casos novos diagnosticados no período de 2001 a 2007 em Cáceres (MT), concluiu que foram diagnosticados 34 casos novos de hanseníase, dos quais 26 casos $(76,5 \%)$ eram paucibacilares e 8 casos $(23,5 \%)$ eram multibacilares. Verificou-se que dos casos paucibacilares diagnosticados, 55,9\% (19 casos) eram representados pelo gênero feminino, enquanto aqueles diagnosticados como multibacilares 17,6\% (6 casos) eram representados pelo gênero masculino. Observou-se também que 35,3\% dos casos novos diagnosticados eram contatos domiciliares ou de vizinhança e, para cada seis casos novos diagnosticados, um contato apresentou hanseníase (GARCIA et al., 2013).

Durante a execução do projeto, também observou-se maior frequência de participantes com faixa etária entre 5-12 anos de idade, com 45,8\%, seguido pela faixa etária entre 13-19 anos, com 23,7\% de participação. Pessoas de 60 anos ou mais apareceram em terceiro lugar, com 9,3\% de participação; entre 40-49 anos, após, com 6,1\% de participação. A faixa etária entre 30-39 apresentou 5,2\% de participação; entre 50-59, com 4,8\% e entre 20-29, com 4,3\% de participação. A faixa etária com menor índice de participação foi de menores de 5 anos, 0,7\% de participação (Figura 2). 
Educando para o diagnóstico precoce da hanseníase no município de Rondonópolis- Mato Grosso

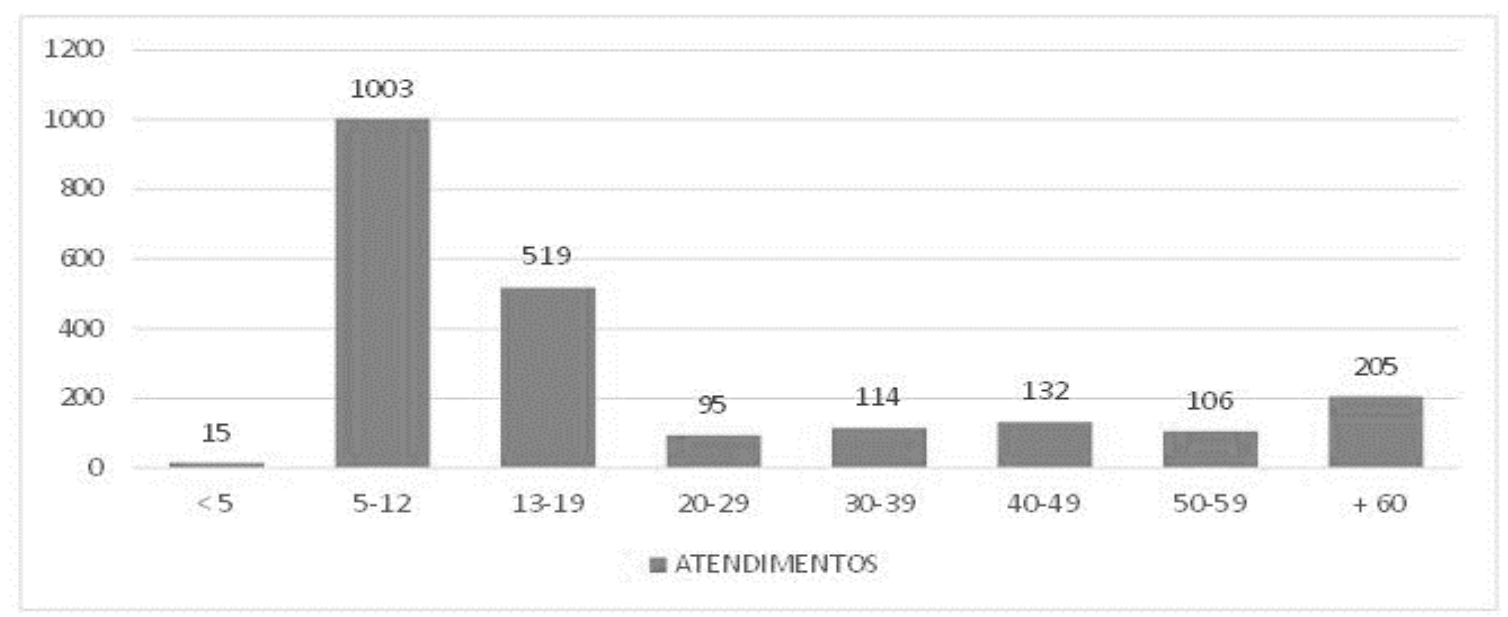

Figura 2: Atendimentos realizados pelo projeto de Extensão Hanseníase: diagnóstico precoce é a solução, de acordo com a faixa etária. Rondonópolis (MT), 2015.

Esses dados em relação à faixa etária, justificam-se devido à realização do evento "Semana da Pátria" realizada na Escola Estadual Santo Antônio, com total de 947 atendimentos. Os alunos da escola enquadravam-se entre as faixas etárias 5-12 anos ou 13-19 anos, aumentando o índice de participação desse público.

Em Rondonópolis (MT) no ano de 2012, foram atendidas 1.483 pessoas neste projeto de extensão, sendo detectados 31 casos suspeitos de hanseníase. Destes, o maior número de casos foi entre o sexo feminino (51,61\%), já em relação à faixa etária foram detectados casos em todas as faixas acima de 5 anos, sendo que houve maior incidência entre as faixas de 5-12 anos e 40-49 anos; ambos com 22,58 \%, sendo que entre os menores de 5 anos não foram detectados casos suspeitos (SANTOS et al., 2013).

A hanseníase pode atingir todas as idades, de ambos os sexos; ocorre raramente em crianças e há uma incidência maior em homens do que nas mulheres, na maioria das partes do mundo (BRASIL, 2002). Segundo a Organização Mundial de Saúde (OMS), foram notificados 20.648 casos novos de hanseníase em menores de 15 anos no mundo. Esse número representou 9\% (20.648 / 228.474) dos casos detectados em 2010. No mesmo ano foram notificados 2.461 casos novos de hanseníase nessa faixa etária, no Brasil. O Brasil ocupa o segundo lugar do mundo em número de casos detectados, ficando atrás da Índia. Em 2010, o relatório anual da OMS revelou uma proporção de 37\% (85.285 / 228.474) de mulheres entre os casos detectados no mundo. No Brasil, o percentual do gênero feminino foi de 45\% (15.513 / 34.894) (WHO, 2011).

Uma estratégia para a redução e para o alcance da meta de eliminação dos casos de hanseníase é o diagnóstico precoce e a cura dos casos diagnosticados. A hanseníase não está distribuída de forma homogênea no Brasil. Segundo o DATASUS EM 2011, os estados do 
Educando para o diagnóstico precoce da hanseníase no município de Rondonópolis- Mato Grosso

Maranhão, Mato Grosso, Pará e Rondônia são os que concentram maior endemicidade da doença (BRASIL. 2012).

Entre os anos de 1999 a 2010, foi estimada uma diminuição na prevalência da hanseníase em mais de 148 países ou territórios de acordo com a OMS. Faz-se necessária que seja implantada estratégia de controle da transmissão da hanseníase, principalmente, nos países em desenvolvimento (PENNA \& PENNA, 2012).

O coeficiente de prevalência de hanseníase do país no ano de 2010 era equivalente a 1,56 casos/10 mil hab., representando diminuição de 8\% quando comparado ao valor obtido em 2004 (1,7 casos/10 mil hab.). Entre os estados que apresentam coeficiente de prevalência alto (entre 5 e 9,99 casos/10 mil habitantes) estão o Mato Grosso e o Maranhão (IGNOTTI; PAULA, 2011; BRASIL,2012).

No Estado do Mato Grosso foi detectada a doença em 1960, consequentemente mostrando um crescimento exponencial entre 1970 a 1995 a partir daí se mantendo estabilizado num elevado índice. Esse aumento foi em consequência do crescimento econômico com a abertura da BR Santarém-Cuiabá que resultou no grande crescimento ficando inclusive maior que a média nacional, consequentemente nesta mesma época houve também um aumento no percentual de notificação de casos de hanseníase (MAGALHÃES et al.,2011). O município de Rondonópolis situado em Mato Grosso apresentou 1.919 casos de Hanseníase notificados e confirmados entre aos anos de 2001 a 2010. Somente em 2010 o município apresentou 198casos novos da patologia (CRUZ NETO; SOUZA, 2012; MATO GROSSO, 2011).

As novas entradas por recidiva de hanseníase (323 casos) foram confirmadas em $80 \%$ nas unidades básicas de saúde e em 20\% nas unidades especializadas no estado de Mato Grosso (municípios de Cuiabá, Cáceres, Diamantino, Rondonópolis ou Várzea Grande) nos anos de 2004 a 2006. O sexo masculino atingiu 71\%, com idade média de 43 anos, não sendo observada diferença nos percentuais das entradas entre as unidades de saúde segundo forma clínica, classificação operacional e grau de incapacidade física da doença. Do total de municípios do estado, 64,7\% apresentou recidiva, com percentual entre $6 \%$ e $20 \%$ de todas as entradas (FERREIRA et al., 2010).

Entre os fatores associados à ocorrência de recidiva da hanseníase neste mesmo estado, incluem: indivíduos residentes em casas alugadas, em domicílio de madeira/taipa, que moram com mais de cinco pessoas, com transtorno de uso de álcool, irregularidade do tratamento, sem esclarecimento sobre a doença/tratamento, que usavam transporte coletivo para o acesso à unidade de saúde, forma clínica da doença e esquema terapêutico. Por outro lado, não estiveram associadas à recidiva: renda mensal individual e familiar, atividade profissional fora do domicílio e Extensio: R. Eletr. de Extensão, ISSN 1807-0221 Florianópolis, v. 13, n. 23, p.45-61, 2016. 
Educando para o diagnóstico precoce da hanseníase no município de Rondonópolis- Mato Grosso

escoamento sanitário; faixa etária, escolaridade, estado civil, tabagismo e número de cigarros consumidos; recebeu visita domiciliar; número; tipo e local de lesões hansênicas, espessamento neural, estado reacional, efeito colateral, grau de incapacidade física no diagnóstico, baciloscopia, valores logaritmos de baciloscopia, biópsia cutânea, tipo e contato intra-domiciliar com casos de hanseníase, comorbidade/situação concomitante, tipos de comorbidades e hospitalização (FERREIRA et al., 2011).

No Estado de Tocantins, entre 2001 a 2012, foram registrados 14.532 casos novos de hanseníase, sendo que o coeficiente médio anual de detecção de casos novos na população geral foi de 93,3 casos/100 mil habitantes. Na população menor de 15 anos, foi de 24,1 casos/100 mil habitantes e foram diagnosticados 4,2 casos com grau 2 de incapacidade física/100 mil habitantes. Estes casos refletem que a transmissão da doença é ativano município, com elevados indicadores, ampla distribuição geográfica e diferenças regionais importantes (MONTEIRO et al., 2015).

Ainda em um estudo descritivo com dados dos casos de hanseníase notificados no estado de Rondônia durante o período de 2001 a 2012, foram notificados 15.648 casos de hanseníase, representando uma média de 1.304 casos ao ano. Nestes anos, houve diminuição de 33,8\% na incidência da hanseníase no estado, de 91,5 casos novos por 100 mil habitantes em 2001 para 60,6 casos novos por 100 mil habitantes em 2012. A maior parte dos casos foi notificada em homens $(57,1 \%)$ e indivíduos de cor da pele parda (46,1\%). A forma clínica indeterminada da doença foi observada em 2.728 (17,8\%) casos, 4.462 (29,1\%) foram classificados com a forma tuberculoide, $6.437(42,2 \%)$ com a forma dimorfa e $1.673(10,9 \%)$ com a virchowiana. A maioria dos casos não apresentava incapacidades (grau 0: 75\%), enquanto 46,6\% foram classificados como paucibacilares (VIEIRA et al., 2014).

O município de Duque de Caxias (RJ) passou por mudanças acentuadas no quadro epidemiológico entre 1998 e 2006, relacionadas, principalmente, à detecção precoce dos casos de hanseníase. Elevação e posterior redução da taxa de detecção foram vistas no período, associadas a uma redução dos casos em mulheres, além da descentralização do atendimento para todas as unidades de saúde, para o diagnóstico precoce e o menor risco de desenvolvimento de incapacidades físicas, assim como a informação, educação e comunicação sobre a hanseníase (DUARTE-CUNHA et al., 2012).

Além disso, durante o desenvolvimento deste projeto, foram detectados 22 casos suspeitos de hanseníase, sendo verificado maior índice destes casos no evento realizado na Praça Brasil "Dia Mundial da Hanseníase”, com detecção de 08 pessoas. No Centro de Saúde Nossa Senhora do Amparo, nas ESF Cidade de Deus e Vila Paulista apontaram 03 casos suspeitos em Extensio: R. Eletr. de Extensão, ISSN 1807-0221 Florianópolis, v. 13, n. 23, p.45-61, 2016 
Educando para o diagnóstico precoce da hanseníase no município de Rondonópolis- Mato Grosso

cada evento. Na "Ação Global" foram encontrados 02 casos suspeitos. Durante os eventos realizados na ESF Parque Industrial e "Casa Esperança" foi detectado apenas 01 caso em cada unidade. Já no "Mutirão de exames de pele e tuberculose no "Lar Cristão", "Posto Masut" e ESF Cidade Alta não foram encontrados casos suspeitos de hanseníase (Figura 3).

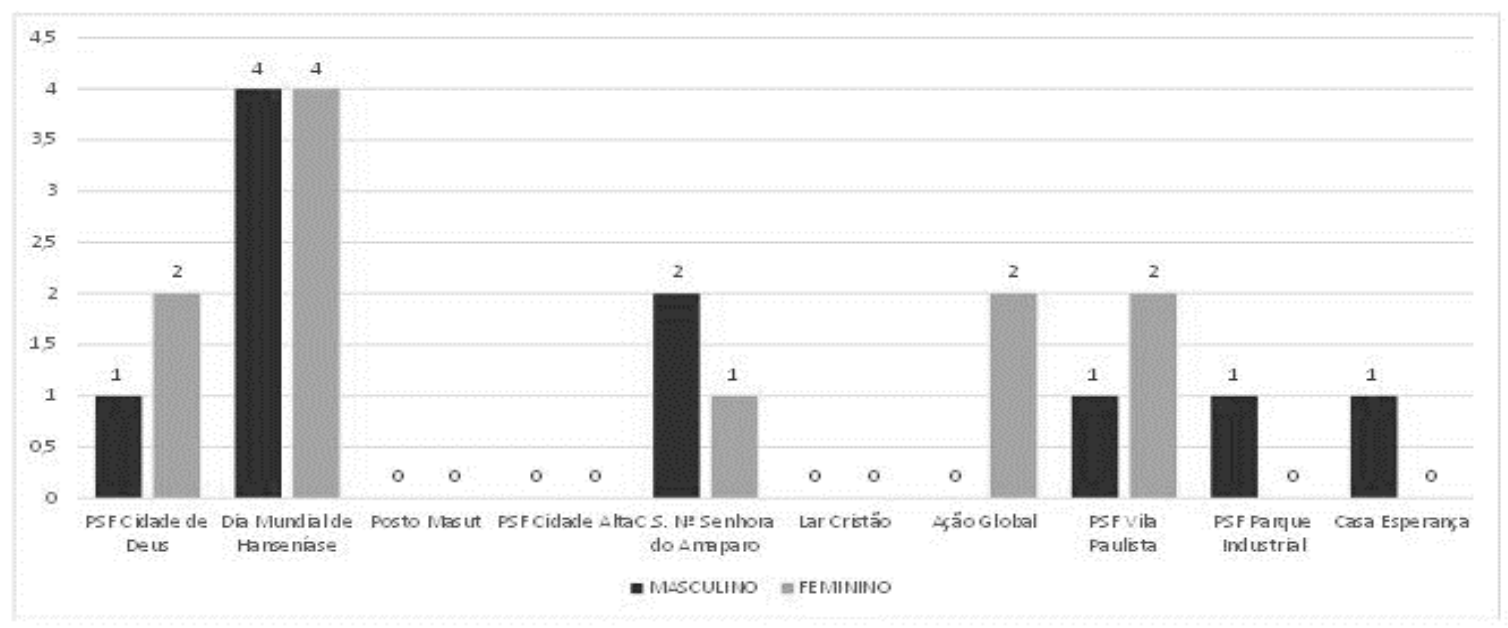

Figura 3: Distribuição dos casos suspeitos de hanseníase detectados durante as ações do projeto de Extensão Hanseníase: diagnóstico precoce é a solução. Rondonópolis (MT), 2015.

Apesar desta incidência ser alta em Rondonópolis, em contraposição com os dados deste projeto, em cidades do Paraná, entre os anos de 2001 a 2010, foi registrado um total de 2.605 casos novos de hanseníase em três municípios (882 em Foz do Iguaçu; 1.054 em Curitiba e 669 em Londrina). Notou-se uma redução do coeficiente de prevalência da hanseníase por 10.000 habitantes em Foz do Iguaçu (de 4,3 para 2,6) e Londrina (de 1,7 para 1,2); Curitiba apresentou menos de 1 caso por 10.000 habitantes no período; a proporção de cura e consequente efetividade do tratamento mostrou-se regular nestes três municípios (OLIVEIRA et al., 2015).

Além do mais, em relação aos casos suspeitos, foi verificado maior número de casos entre o sexo feminino 11 pessoas $(52,4 \%)$ e o sexo masculino 10 pessoas (47,6\%). Em relação à faixa etária foram detectados casos em todas as faixas acima de 5 anos, sendo que houve maior incidências entre as faixas de 40-49 anos com 33,30\%, em seguida igual ou maiores de 60 anos com 28,60\%, as faixas etárias de 30-39 anos e 50-59 anos aparecem iguais com 14,30\% seguido por 20-29 anos com 9,50\%. Entre os menores de 5 anos não foram detectados casos suspeitos (Figura 4). 
Educando para o diagnóstico precoce da hanseníase no município de Rondonópolis- Mato Grosso

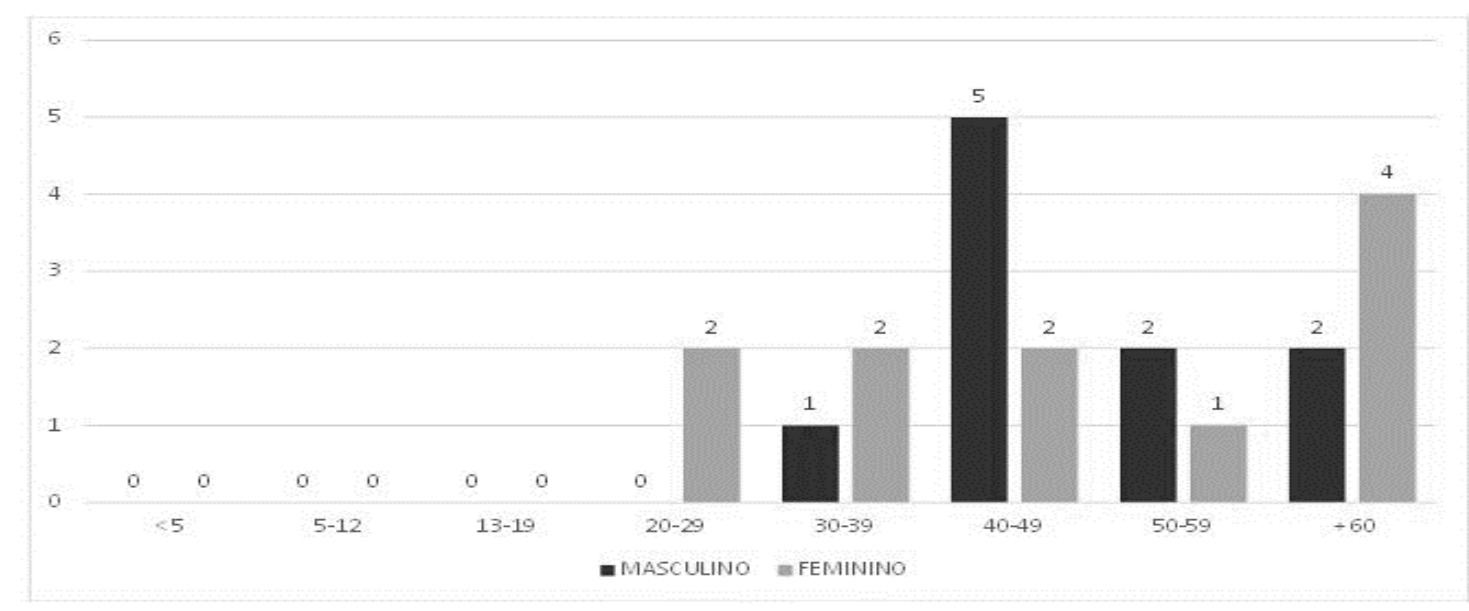

Figura 4: Distribuição dos casos suspeitos de hanseníase detectados durante as ações do projeto de Extensão Hanseníase: diagnóstico precoce é a solução, de acordo com a faixa etária. Rondonópolis (MT), 2015.

O controle da hanseníase perpassa pela interrupção da transmissão e pela redução da incidência, cabendo aos gestores de saúde a necessidade de conservar as atividades de controle da hanseníase para ampliar programas de controle no futuro. A fim de reduzir a prevalência oculta da doença, deve-se promover a detecção de casos, que, por sua vez, implica no aumento da prevalência conhecida (TALHARI et al., 2012).

Faz-se indispensável que a equipe interdisciplinar atenda os casos de hanseníase de forma humanizada e integral. Lanza \& Lana (2011) descrevem que em Almenara (MG), entre 2007 e 2008, os médicos mantêm uma assistência curativa e individual; os enfermeiros dominam todo o processo de trabalho em hanseníase; e os agentes comunitários de saúde realizam as ações educativas, a busca de suspeitos dermatológicos, de contatos e de faltosos. Cabe a gestão pública, incentivar a realização de ações de controle da hanseníase de acordo com as normas técnicas e protocolos do Ministério da Saúde.

Desta maneira este projeto de extensão contribuiu para o diagnóstico precoce da hanseníase no município em estudo. A universidade vai até a comunidade, ou por vezes, pode receber pessoas da comunidade em seu campus, prestando-lhes serviços, assistência, auscultando lhes os anseios e as necessidades. Portanto, ensino, pesquisa e extensão são atividades interdependentes, complementares e precisam ter valorações equivalentes no sistema universitário. A qualidade e o sucesso dos profissionais formados pelas universidades, dependem, diretamente, do nível de desenvolvimento, equilíbrio e harmonia entre essas três áreas da Universidade (SILVA, 1997). 
Educando para o diagnóstico precoce da hanseníase no município de Rondonópolis- Mato Grosso

\section{CONSIDERAÇÕES FINAIS}

Através do desenvolvimento deste projeto de extensão em parceria com a SMS, foram desenvolvidas diversas ações de saúde que envolveram outros acadêmicos da UFMT e diversos profissionais de saúde atuantes nas unidades básicas de saúde do município e das escolas, havendo uma integração importante entre os setores da comunidade. Além disso, houve uma relevante importância para o ensino da graduação e para a população deste município a quem foi oferecido maior conhecimento sobre a necessidade de prevenção da hanseníase.

Neste artigo, conclui-se que os objetivos do projeto de extensão foram alcançados e com a confirmação dos casos de hanseníase, os usuários foram encaminhados ao serviço de referência para tratamento e não disseminação da hanseníase, fato que melhora a qualidade da assistência à saúde prestada por este município. Cabe ressaltar que nas avaliações realizadas pelo público-alvo, as ações do projeto foram reconhecidas com excelência pela população, pelos alunos, professores, coordenadores e diretores das escolas e pelos profissionais de saúde envolvidos.

Através da educação em saúde, este projeto considerado como uma ação de cidadania, além de uma extensão universitária, colaborou significativamente com uma melhor qualidade de assistência à saúde para a população, orientando e promovendo o diagnóstico precoce de hanseníase neste município. Sugerem-se novos projetos e pesquisascientíficas relacionados a esta temática, a fim de que possam favorecer a qualidade da atenção básica de saúde, estimulando a participação da academia através da extensão.

\section{REFERÊNCIAS}

BRASIL. Ministério da Saúde. Secretaria de Políticas de Saúde. Departamento de Atenção Básica.

Guia para o Controle da hanseníase. Brasília: Ministério da Saúde, 2002.

Ministério da Saúde. Secretaria de Vigilância em Saúde. Departamento de Vigilância Epidemiológica. Vigilância em saúde: situação epidemiológica da hanseníase no Brasil. Brasília: Ministério da Saúde, 2008a.

Ministério da Saúde. Secretaria de Vigilância em Saúde. Departamento de Vigilância Epidemiológica. Hanseníase e direitos humanos: direitos e deveres dos usuários do SUS. Brasília: Ministério da Saúde, 2008b. 
Educando para o diagnóstico precoce da hanseníase no município de Rondonópolis- Mato Grosso

Ministério da Saúde. Secretaria de Vigilância em Saúde. Hanseníase: guia de Vigilância Epidemiológica. 7.ed. Brasília: Ministério da Saúde, 2009.

Ministério da Saúde. Secretaria de Vigilância em Saúde. Departamento de Vigilância Epidemiológica. Doenças infecciosas e parasitárias: guia de bolso. 8.ed. rev. Brasília: Ministério da Saúde, 2010.

Ministério da Saúde. Secretaria de Vigilância em Saúde. Situação Epidemiológica Hanseníase Brasil 2011, Brasília. 2012.

CRUZ NETO, L.R.; SOUZA, T.J. Caracterização dos casos novos de Hanseníase notificados em Rondonópolis. 2012. Trabalho de Conclusão do Curso de Enfermagem. UFMT/ICEN/CUR.

DUARTE-CUNHA, M.; SOUZA-SANTOS, R.; MATOS, H.J.; OLIVEIRA, M.L.W. Aspectos epidemiológicos da hanseníase: uma abordagem espacial. Cad. Saúde Pública, v.28, n.6, p.11431155, 2012.

FERREIRA, S.M.B.; IGNOTTI, E.; SENIGALIA, L.M.; SILVA, D.R.X.; GAMBA, M.A. Recidivas de casos de hanseníase no estado de Mato Grosso. Rev Saúde Pública, v.44, n.4, p.650-7, 2010.

FERREIRA, S.M.B.; IGNOT'TI, E.; SENIGALIA, L.M.; SILVA, D.R.X.; GAMBA, M.A. Fatores associados à recidiva em hanseníase em Mato Grosso. Rev Saúde Pública, v.45, n.4, p.756-64, 2011.

GARCIA，D.R.; IGNOTTTI, E.; CORTELA, D.C.B.; XAVIER, D.R.; BARELLI, C.S.G.A. Análise espacial dos casos de hanseníase, com enfoque à área de risco, em uma unidade básica de saúde no município de Cáceres (MT). Cad. Saúde Colet., v.21, n.2, p.168-72, 2013.

GOVERNO MUNICIPAL DE RONDONÓPOLIS. Secretária Municipal de Saúde. Protocolo de Atendimento a hanseníase na cidade de Rondonópolis - MT. 2011. Disponível em: $<$ http://www.rondonopolis.mt.gov.br/docs/Protocolo_Hanseniase_para_impress_o.pdf>. 
Educando para o diagnóstico precoce da hanseníase no município de Rondonópolis- Mato Grosso

IGNOTTI, E.; PAULA, R.C. Situação epidemiológica da hanseníase no Brasil: análise de indicadores selecionados no período de 2001 a 2010. Secretaria de Vigilância em Saúde, Ministério da Saúde, 2011.

LANZA, F.M.; LANA, F.C.F. O processo de trabalho em hanseníase: tecnologias e atuação da equipe de saúde da família. Texto Contexto Enferm, v.20, (esp), p.238-46, 2011.

MAGALHÃES, M.C.C.; SANTOS, E.S.S.; QUEIROZ, M.L.; LIMA, M.L.; BORGES, R.C.M.; SOUZA, M.S.; RAMOS, A.N. MigrationandHansen'sdisease in Mato Grosso. Revista brasileira de epidemiologia, v.14, n.3, p.386-397, 2011.

MATO GROSSO. Secretaria de Estado de Saúde. Superintendência de Políticas de Saúde. Coordenadoria de Gestão da Informação em Saúde. Informações Regionais de Saúde ERS Rondonópolis - MT 2010, Cuiabá. 2011. 36 p.

MARTINS, P. V.; CAPONI, S. Hanseníase, exclusão e preconceito: histórias de vida de mulheres em Santa Catarina. Ciências e Saúde Coletiva, v.15, supp.l, p.1047-1054, 2010.

MINISTÉRIO DA SAÚDE. Departamento de Informática do SUS- DATASUS. Disponível em: http://www2.datasus.gov.br/DATASUS/index.php?area=02. Acessoem: 20 jun. 2015.

MONTEIRO, L.D.; ROGERLÂNDIO, F.; MELO, M.; BRITO, A.L.; ALENCAR, C.H.; HEUKELBACH, J. Spatial patterns of leprosy in a hyperendemic state in Northern Brazil, 2001 2012. Rev. Saúde Pública, v.49, n.84, p.1-8, 2015.

OLIVEIRA, K.S.; SOUZA, J.; CAMPOS, R.B.; SILVA-SOBRINHO, R.A. Avaliação dos indicadores epidemiológicos e operacionais para a hanseníase em municípios prioritários no estado do Paraná, 2001 a 2010. Epidemiol. Serv. Saúde, v.24, n.3, p.507-516, 2015.

PENNA, M.L.F.; PENNA, G.O. Leprosy frequency in the world, 1999-2010.Mem. Inst. Oswaldo Cruz, v.107, suppl.1, p.3-12, 2012.

SANTOS, D.A.S.; GONÇALVES, R.E.L.M.; NASCIMENTO, A.M.; CRUZ-NETO, L.R. Hanseníase: diagnóstico precoce é a solução. Participação 23/24, p.133-141, 2013. 
Educando para o diagnóstico precoce da hanseníase no município de Rondonópolis- Mato Grosso

SILVA, M.C.D.; PAZ, E.P.A. Educação em saúde no programa de controle da hanseníase: a vivência da equipe multiprofissional. Esc Anna Nery RevEnferm, v.14, n.2, p.223-229, 2010.

SILVA, O. D. O que é extensão universitária? Integração ensino-pesquisa-extensão. III, n.9, p.148-9, maio, 1997.

TALHARI, S.; GROSSI, M.A.F.; OLIVEIRA, M.L.W.; GONTIJO, B.; TALHARI, C.; PENNA, G.O. Hansen'sdisease: a vanishingdisease?.Mem. Inst. Oswaldo Cruz , v.107, suppl.1, p.13-16, 2012.

VIEIRA, G.D.; ARAGOSO, I.; CARVALHO, R.M.B.; SOUSA, C.M. Hanseníase em Rondônia: incidência e características dos casos notificados, 2001 a 2012. Epidemiol. Serv. Saúde, v.23, n.2, p.269-275, 2014.

WORLD HEALTH ORGANIZATION - WHO. Leprosy update, 2011. Wkly. Epidemiol. Rec., Geneve, v.86, n.36, p.389-399, Sept. 2011. Disponível em: <http://www.who.int/wer/2011/wer8636.pd 